\title{
Innervation of the spinal dura mater
}

\author{
M. A. EDGAR AND S. NUNDY \\ From the Anatomy School, Cambridge
}

The nerve supply to the spinal dura mater has long been a subject of controversy.

Von Luschka (1850), in his classic monograph, described a series of nerves which passed through the intervertebral foramina. Each nerve corresponded with one foramen and traversed it ventral to the spinal nerve. He traced this recurrent nerve by gross dissection and noted that, within the spinal canal, it supplied the bone and blood vessels. Hence it was named the sinu-vertebral nerve (now termed ramus meningeus P.N.A.). Luschka observed that branches of each ramus meningeus remained entirely outside the spinal dura mater although some were seen close to its surface. Other contemporary workers, notably Pürkinje (1845), saw no epidural nerves piercing the dura.

Rüdinger (1857), however, dissected nerves which ran along the ventral surface of the dura mater and then entered it. Some fibres were found to be perivascular, others to pursue an independent course. Further studies by other workers, reviewed by Testut (1930), confirmed these findings. By 1904 the presence of intrinsic nerves of the spinal dura mater was well enough established for Poirier and Charpy to describe them in their textbook. Hovelacque (1925), Pederson, Blunck, and Gardner (1956), and Stilwell (1956), by various methods, including gross dissection and examination of stained serial sections, identified the presence of nerves which penetrated the spinal dura mater.

However, contrary to a century of agreed findings, Bridge (1959) stated definitely that the spinal dura mater, unlike its cranial counterpart (Penfield and McNaughton, 1940), had no intrinsic nerve supply; epidural nerves were seen to end on the dural surface only.

Kimmel's recent study (1961) of Bodian-stained foetal sections, however, demonstrated intrinsic nerve fibres. He concluded that these were only to be seen with difficulty and that the different conclusions reached by previous workers were due to the varying quality of their preparations.

The origin of nerve fibres supplying the dura (whether they are confined to its surface or are truly intrinsic) is generally agreed to be from the mening- eal rami. However, Hilbert (1878) described nerve fibres passing along the ligamenta denticulata from the spinal cord to the internal surface of the dura. In addition, Stilwell (1956) and Kimmel (1961) observed dorsal recurrent nerves supplying the dorsal aspect of the dura mater.

Clarification of the nerve supply to the spinal dura mater is important, especially with regard to the mechanism of dural sensitivity and pain in its clinical application.

\section{METHODS}

The difficulty of distinguishing small nerve filaments from connective tissue strands is well documented,, and it is hard to follow finer ramifications of nerves even in stained serial sections. Roofe (1940) was not convinced that any of the fibrous strands within the intervertebral foramina were in fact nerves. In thiso study, a combination of methods, involving gross dissection and histological examination, has been em ployed in an attempt to circumvent these difficulties and to define the origin, course, and distribution of nerves to the spinal dura mater.

GROSS DISSECTION Two formalin-fixed cadavers (one adult and an 8-month foetus) were dissected to demonstrate the origin and distribution of nervous filaments running through the inter-vertebral foramina. Strands of ambiguous nature were examined histologically after silver impregnation.

In addition four paravertebral blocks of fresh adult post-mortem material and three whole specimens of spinal dura with epidural tissue were similarly dissected after staining in Schiff's reagent (McManus, Saunders, Penton, and Cason, 1950; Bridge, 1959). By this technique finer ramifications of nerves, stained blue, were more readily identified.

HISTOLOGY The dura from seven spinal cords was divided into segments. These were impregnated with silver to stain intrinsic nerve fibres using a modified BielschowskyGross method (Penfield and McNaughton, 1940). Some dural segments were stained by osmic acid to detect myelinated nerve fibres.

Finally an attempt was made to stain nerve endings in spinal dura using intravital methylene blue in two rabbits (Feindel, Sinclair, and Weddell, 1947). 


\section{RESULTS}

The origin, course, and relevant distribution of the meningeal rami were determined. There was considerable variation in the findings at different levels and at certain levels the nerves were asymmetrical on the two sides. There were no significant differences between adult and foetal dissections.

ORIGIN OF RAMUS MENINGEUS Typically a ramus meningeus has a dual origin receiving sympathetic fibres from the related ramus communicans and other fibres from the anterior primary ramus (Fig. 1). The sympathetic contribution is usually multiple with two or three main filaments coming from the ramus communicans. Occasional branches pass from the adjacent sympathetic ganglion or sympathetic trunk.

The spinal component usually consists of one main filament and comes off the anterior primary ramus. At several different levels in the foetal dissection additional nerve roots could be traced from the dorsal root ganglion. In the adult thoracic region the posterior primary ramus was observed to give contributions at several levels.

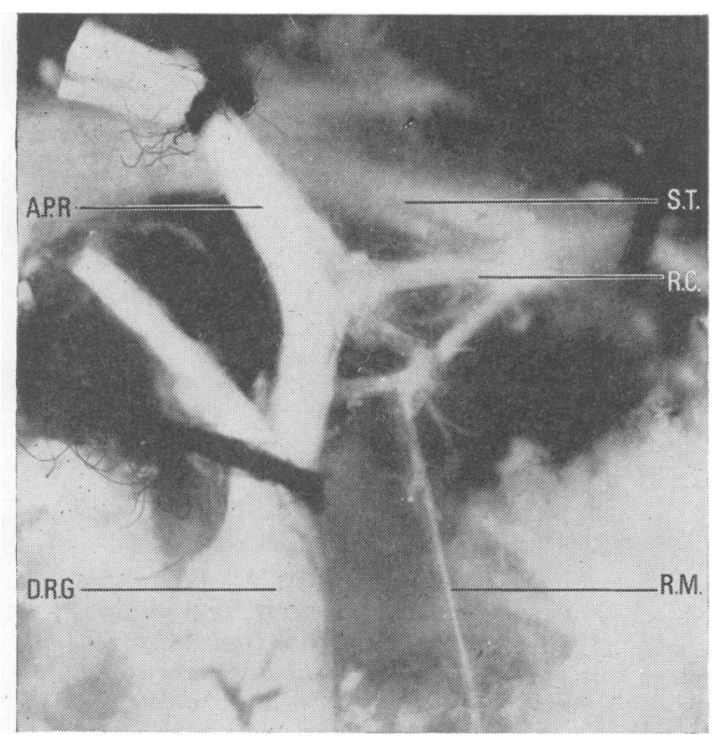

FIG. 1. Paravertebral gross dissection of an 8-month foetus at the level of T10 demonstrating a prominent filament of the ramus meningeus (R.M.). Two finer filaments have broken. The dorsal root ganglion (D.R.G.) and sympathetic trunk (S.T.) are drawn aside by fine thread to gain a better exposure. The two roots of the ramus meningeus are from the ramus communicans $(R . C$. and the anterior primary ramus (A.P.R.). $\times 12$.
COURSE Each 'ramus meningeus' is composed of up to six separate nerve filaments running through an intervertebral foramen. Usually one filament is larger than the others and may be considered as the main ramus.

Within the spinal canal the branches of the ramus meningeus destined for the dura mater follow three main courses:

1 The most prominent ramus passes cranially around the base of the pedicle giving ascending, descending, and transverse branches to a plexus of nerves situated around and within the posterior longitudinal ligament. Here there are anastomoses with similar nerves of adjacent segments and also across the midline. Anterior to the posterior longitudinal ligament this plexus is continuous with that around the basi-vertebral veins. Posteriorly a few branches from this plexus pass directly backwards near the midline to the ventral aspect of the dura mater.

2 A small filament passes directly to the dura mater within the spinal canal, reaching it just ventral to the point at which the spinal nerve root pierces the dura.

3 A third group of fibres passes longitudinally within the epidural tissue. Mostly these are directed caudally although a small number pass cranially. Branches of these epidural nerves reach the dura.

DISTRIBUTION OF NERVES ON THE DURAL SURFACE The ventral surface of the spinal dura mater shows numerous fine nerve trunks running longitudinally on it in parallel. Nerves from adjacent levels overlap. No nerves were observed on the dorsal surface of the dura mater.

Nerves as described under (1) above are few and lie along or adjacent to the midline anteriorly. Nerves as described under (2) above are placed postero-laterally near the line of emerging nerve roots. Nerves as described under (3) above, passing from the epidural tissues, lie in an intermediate position. Each nerve, on reaching the surface of the dura mater, divides into an ascending and descending branch (Fig. 2). The descending division passes caudally one half to two segments of the spinal cord, the other ascends up to one segment.

DISTRIBUTION OF NERVES WITHIN THE DURA MATER During their course the longitudinally running nerve filaments pierce the dural surface and give transverse branches, many of which subdivide within the dural substance. All finer ramifications are intrinsic. This fact was established by assessing focus levels in microscopic examination of stained dural segments, by microdissection, and by examination of selected transverse sections of stained dura. 


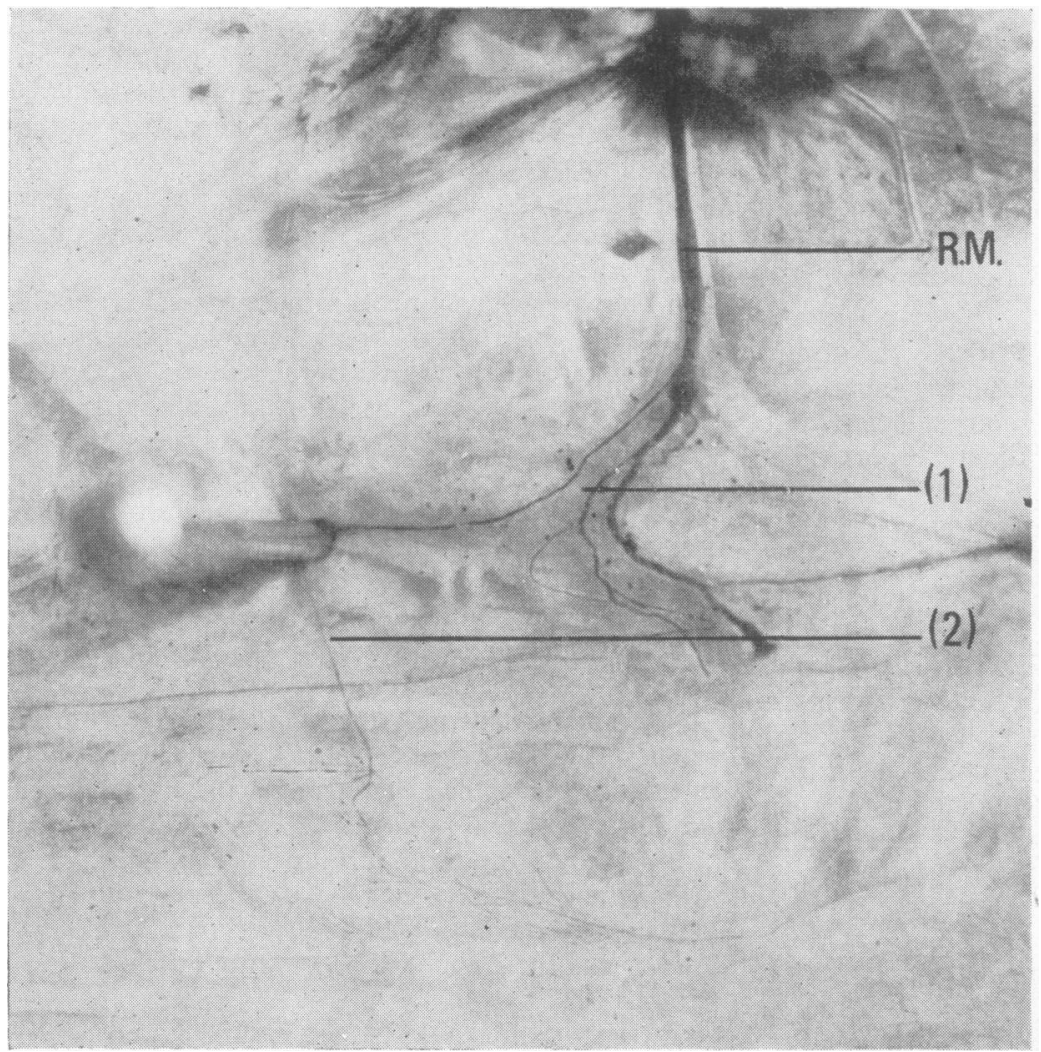

FIG. 2. Filament of a ramus meningeus (R.M.) stained with $S c h i f f$ 's reagent passing to the ventral surface of the dura just medial to the spinal nerve root in the upper lumbar region. (1) Branch to dura dividing into a short ascending and much longer descending filament. (2) Branch to dura (held aside by pin) reaching it more medially after a short epidural course. Here its ascending branch on the dural surface is longer. $\times 8$.

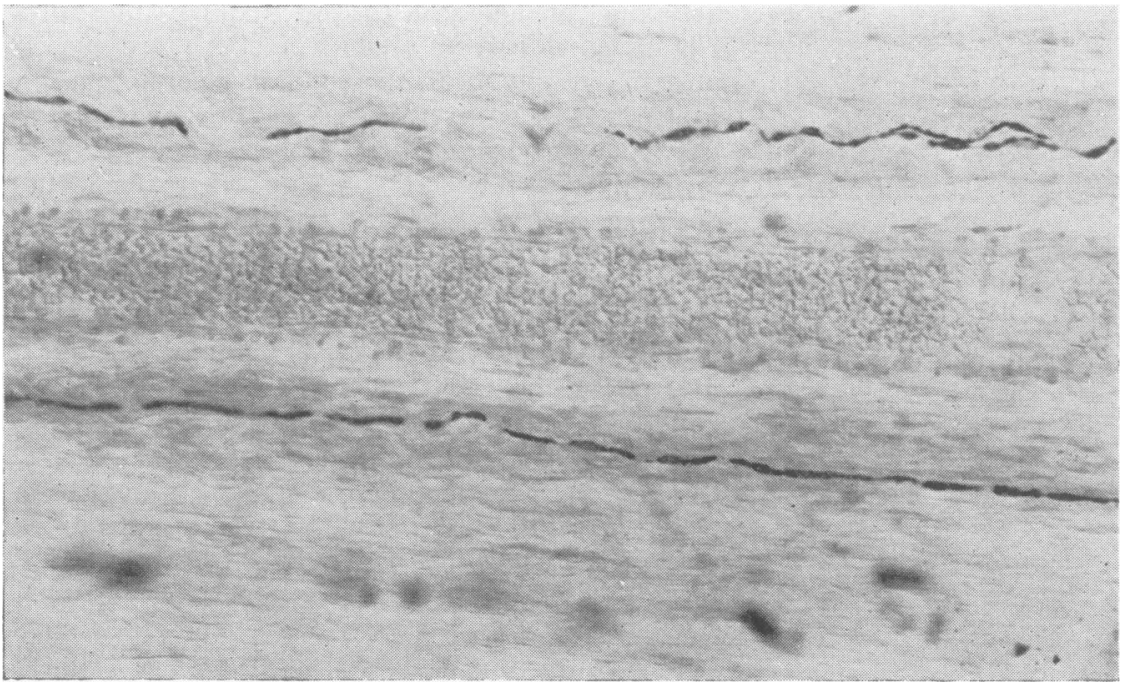

FIG. 3. Perivascular nerve fibres within the dura stained by the Bielschowsky-Gross method. $\times 360$. 
Most fibres accompany blood vessels (Fig. 3), a few run independently. No intrinsic nerve fibres were found in the dorsal part of the dura mater although blood vessels were numerous.

Osmic acid staining revealed some myelinated fibres within the dural substance. No definitive nerve endings were seen in the silver- or methyleneblue-stained material.

\section{DISCUSSION}

An attempt has been made to provide, within the limits of the methods used, an accurate account of the nerve supply to the spinal dural mater. Contrary to the finding of Bridge (1959) the dura has been shown to possess intrinsic nerves derived from the meningeal rami. Luschka's observation that each ramus meningeus has a dual origin from the adjacent grey ramus communicans and anterior primary ramus has been confirmed. These contributions to the ramus meningeus are very variable (Wiberg, 1949) and, as stressed by Hovelacque (1925), usually multiple.

Our results agree with those of Kimmel in establishing that a ramus meningeus is not a single trunk but a series of perivascular filaments running through an intervertebral foramen.

Nerves to the dura mater may be considered to follow three main courses. Bridge (1959) described the main ramus running towards the posterior longitudinal ligament and Pedersen et al. (1956) represent its course adjacent to the pedicle by means of a diagram. Luschka (1863) shows the anastomoses between adjacent rami in a drawing of a dissection. In this study, these nerves have also been seen and noted as giving rise to dural branches considered under (1) above. Stilwell observed a nerve passing to the dura mater immediately within the spinal canal. This was derived from the ramus meningeus and lay just ventral to the spinal nerve. These fibres correspond to those of (2). The nerve filaments of (3) have not been specifically described in previous studies.

Differences of opinion exist in the literature concerning the segmental distribution of each ramus meningeus. Spurling and Bradford (1939) published a diagram of a dissection by Roofe showing the meningeal branch of the second lumbar nerve descending two and a half segments to supply the posterior longitudinal ligament. Millen and Woollam (1962) suggest that the pattern of innervation of the dura may be similar. Other workers, however, have observed that each ramus meningeus divides into an ascending and descending branch. Pedersen et al. found that both these branches supplied the dura in the lumbar region, but were uncertain as to the extent of distribution. From their point of origin Kimmel describes longer descending nerves in the lumbar region and longer ascending nerves in the cervical region. In the present study a considerable degree of overlap of meningeal nerves derived from adjacent segments was found, and the general pattern appears to be that fibres derived from a ramus meningeus are distributed to the segment above and to two segments below. This pattern appears to hold true for the cervical region as well.

The nerves supplying the spinal dura mater all appear to be derived from meningeal rami. Although they were specifically sought, no nerves were found passing along the ligamenta denticulata to the internal surface of the dura. No nerves were observed in the substance or on the surface of the dorsal dura mater. The dorsal recurrent branches described by Kimmel and Stilwell do not supply the dura mater.

The nature of the nerve fibres concerned is a subject which is open to speculation. It seems likely that many subserve a pain function. This is consistent both with their small size and with the presence of naked nerve endings. The sympathetic root of the ramus meningeus may also transmit visceral afferent fibres (Mitchell, 1953).

The above findings have a double interest. First they explain the fact that no pain is elicited on piercing the dorsal part of the spinal dura mater in procedures such as lumbar puncture (Cyriax, 1962). Smyth and Wright (1958) concluded, as a result of experiments in which tension was put on nylon loops placed through the spiral dura, that it is almost insensitive. This finding may be explained if, in their experiment, the traction was applied solely to the non-innervated dorsal region.

Secondly, the wide distribution of back pain after the protrusion of a single intervertebral disc may be explained by pressure on or traction of the dura (Cyriax, 1945). Our findings have shown that the dura has a considerable overlap in supply from nerve fibres of adjacent levels. Involvement of an individual ramus meningeus in protrusion of an intervertebral disc has been discussed by Kaplan (1947).

\section{SUMMARY}

The spinal dura mater has been shown to possess an intrinsic nerve supply. These nerve fibres are all derived from the meningeal rami and reach the ventral dural surface by three main courses. Here the nerves divide into ascending and usually longer descending filaments which run longitudinally in parallel on the dural surface. A considerable overlap of nerve fibres from adjacent segments was noted 
in all regions. Finer filaments penetrate the dural substance where they subdivide. No definitive nerve endings were found. No nerves were traced to the dorsal aspect of the spinal dura mater.

Our observations explain the absence of dural pain on lumbar puncture and may clarify the wide distribution of back pain which is often found following protrusion of a single intervertebral disc.

We wish to express our thanks to Professor J. D. Boyd for his advice and encouragement and to Dr. D. H. M. Woollam who suggested this study. We are also grateful to J. W. Cash and J. F. Crane for technical and photographic assistance and to Mr. W. Simons for help with translation.

\section{REFERENCES}

Bridge, C. J. (1959). Innervation of spinal meninges and epidural structures. Anat. Rec., 133, 533-563.

Cyriax, J. H. (1945). Lumbago-Mechanism of dural pain. Lancet, 2, 427-429.

- (1962). Textbook of Orthopaedic Medicine, 4th ed. Vol. 1, pp. 393-395. Cassell, London.

Feindel, W. H., Sinclair, D. C., and Weddell, G. (1947). A new method for investigating the nervous system. Brain, 70, 495-506.

Hilbert, R. (1878). Zur Kenntnis der Spinalnerven. Thesis, Königsberg. Hovelacque, A. (1925). Le nerf sinu-vertébral. Ann. Anat. path. med Chir., 2, 435-443.

Kaplan, E. B. (1947). Recurrent meningeal branch of the spinal nerves. Bull. Hosp. Jt Dis. (N.Y.), 8, 108-109.
Kimmel, D. L. (1961). Innervation of spinal dura mater and dura mater of the posterior cranial fossa. Neurology (Minneap.), 11, 800-809.

Luschka, H. von. (1850). Die Nerven des menschlichen Wirbelkanales. Tübingen.

- (1863). Die Anatomie der Brust des Menschen. p. 71. Tübingen.

McManus, J. F. A., Saunders, J. C., Penton, G. B., and Cason, J. E (1950). A hitherto undescribed coloring reaction of certain human nerve fibers. Science, 111, 155.

Millen, J. W. and Woollam, D. H. M. (1962). The Anatomy of the Cerebrospinal Fluid, pp. 136-137. Oxford University Press, London.

Mitchell, G. A. G. (1953). Anatomy of the Autonomic Nervous System. p. 17. Livingstone, Edinburgh and London.

Pedersen, H. E., Blunck, C. F. J., and Gardner, E. (1956). The anatomy of lumbosacral posterior rami and meningeal branches of spinal nerves (sinu-vertebral nerves). J. Bone $J$ Surg., 38A, 377-391.

Penfield, W. and McNaughton, F. (1940). Dural headache and innervation of the dura mater. Arch. Neurol. Psychiat.(Chic.), 44, 43-75.

Poirier, P., and Charpy, A. (1904). In Traité d'Anatomie humaine, Vol III, part 3, 2nd ed. pp. 814-816. Masson, Paris.

Purkinje, J. E. (1845). Mikroskopisch-neurologische Beobachtungen Arch. Anat. Physiol. Wiss. Med. (Berl.), pp. 281-295.

Roofe, P. G. (1940). Innervation of annulus fibrosus and posterio longitudinal ligament. Arch. Neurol. Psychiat. (Chic.), 44, $100-103$.

Rüdinger, N. (1857). Die Gelenknerven des menschlichen Körpers. Ferdinand Enke, Erlangen.

Smyth, M. J. and Wright, V. (1958). Sciatica and the intervertebra disc-an experimental study. J. Bone Jt Surg., 40A, 1401-1418.

Spurling, R. G. and Bradford, F. K. (1939). Neurologic aspects of herniated nucleus pulposus. J. Amer. med. Ass., 113, 2019-2022

Stilwell, D. L. Jnr. (1956). The nerve supply of the vertebral columnand its associated structures in the monkey. Anat. Rec., 125 $\mathrm{O}$ 139-162.

Testut, L. (1930). In Traité d'Anatomie humaine, 8th ed. Vol. 3, p. 20 Doin, Paris.

Wiberg, G. (1949). Back pain in relation to the nerve supply of the intervertebral disc. Acta orthop. scand., 19, 211-221. 